\title{
spikeslab: Prediction and Variable Selection Using Spike and Slab Regression
}

\author{
by Hemant Ishwaran, Udaya B. Kogalur and J. Sunil Rao
}

\begin{abstract}
Weighted generalized ridge regression offers unique advantages in correlated highdimensional problems. Such estimators can be efficiently computed using Bayesian spike and slab models and are effective for prediction. For sparse variable selection, a generalization of the elastic net can be used in tandem with these Bayesian estimates. In this article, we describe the R-software package spikeslab for implementing this new spike and slab prediction and variable selection methodology.
\end{abstract}

The expression spike and slab, originally coined by Mitchell and Beauchamp (1988), refers to a type of prior used for the regression coefficients in linear regression models (see also Lempers (1971)). In Mitchell and Beauchamp (1988), this prior assumed that the regression coefficients were mutually independent with a two-point mixture distribution made up of a uniform flat distribution (the slab) and a degenerate distribution at zero (the spike). In George and McCulloch (1993) a different prior for the regression coefficient was used. This involved a scale (variance) mixture of two normal distributions. In particular, the use of a normal prior was instrumental in facilitating efficient Gibbs sampling of the posterior. This made spike and slab variable selection computationally attractive and heavily popularized the method.

As pointed out in Ishwaran and Rao (2005), normal-scale mixture priors, such as those used in George and McCulloch (1993), constitute a wide class of models termed spike and slab models. Spike and slab models were extended to the class of rescaled spike and slab models (Ishwaran and Rao, 2005). Rescaling was shown to induce a nonvanishing penalization effect, and when used in tandem with a continuous bimodal prior, confers useful model selection properties for the posterior mean of the regression coefficients (Ishwaran and Rao, 2005, 2010).

Recently, Ishwaran and Rao (2010) considered the geometry of generalized ridge regression (GRR), a method introduced by Hoerl and Kennard to overcome ill-conditioned regression settings (Hoerl and Kennard, 1970a,b). This analysis showed that GRR possesses unique advantages in high-dimensional correlated settings, and that weighted GRR (WGRR) regression, a generalization of GRR, is potentially even more effective. Noting that the posterior mean of the regression coefficients from a rescaled spike and slab model is a type of WGRR estimator, they showed that this WGRR estimator, referred to as the Bayesian model averaged (BMA) estimator, when coupled with dimension reduction, yielded low testset mean-squared-error when compared to the elastic net (Zou and Hastie, 2005).

Additionally, Ishwaran and Rao (2010) introduced a generalization of the elastic net, which they coined the gnet (short for generalized elastic net). The gnet is the solution to a least-squares penalization problem in which the penalization involves an overall $\ell_{1}$-regularization parameter (used to impose sparsity) and a unique $\ell_{2}$-regularization parameter for each variable (these latter parameters being introduced to combat multicollinearity). To calculate the gnet, a lasso-type optimization is used by fixing the $\ell_{2}$-regularization parameters at values determined by finding the closest GRR to the BMA. Like the BMA, the gnet is highly effective for prediction. However, unlike the BMA, which is obtained by model averaging, and therefore often contains many small coefficient values, the gnet is much sparser, making it more attractive for variable selection.

The gnet and BMA estimators represent attractive solutions for modern day high-dimensional data settings. These problems often involve correlated variables, in part due to the nature of the data, and in part due to an artifact of the dimensionality [see Cai and Lv (2007); Fan and Lv (2008) for a detailed discussion about high-dimensional correlation]. The BMA is attractive because it addresses the issue of correlation by drawing upon the properties of WGRR estimation, a strength of the Bayesian approach, while the gnet achieves sparse variable selection by drawing upon the principle of soft-thresholding, a powerful frequentist regularization concept.

Because high-dimensional data is becoming increasingly common, it would be valuable to have user friendly software for computing the gnet and BMA estimator. With this in mind, we have developed an R package spikeslab for implementing this methodology (Ishwaran, Kogalur and Rao, 2010).

The main purpose of this article is to describe this package. Because this new spike and slab approach may be unfamiliar to users in the R-community, we start by giving a brief high-level description of the algorithm [for further details readers should however consult Ishwaran and Rao (2010)]. We then highlight some of the package's key features, illustrating its use in both low- and high-dimensional settings. 


\section{The spike and slab algorithm}

The spikeslab $\mathrm{R}$ package implements the rescaled spike and slab algorithm described in Ishwaran and Rao (2010). This algorithm involves three key steps:

1. Filtering (dimension reduction).

2. Model Averaging (BMA).

3. Variable Selection (gnet).

Step 1 filters all but the top $n F$ variables, where $n$ is the sample size and $F>0$ is the user specified fraction. Variables are ordered on the basis of their absolute posterior mean coefficient value, where the posterior mean is calculated using Gibbs sampling applied to an approximate rescaled spike and slab posterior. Below is a toy-illustration of how filtering works $[p$ is the total number of variables and $\left(\mathrm{V}_{(k)}\right)_{k=1}^{p}$ are the ordered variables]:

$$
\underbrace{\mathrm{V}_{(1)}, \ldots, \mathrm{V}_{([n F])}}_{\text {retain these variables }} \quad \underbrace{\mathrm{V}_{([n F]+1)}, \ldots, \mathrm{V}_{(p)}}_{\text {filter these variables }} .
$$

The value for $F$ is set using the option bigp.smalln. factor, which by default is set to the value $F=1$. The use of an approximate posterior in the filtering step is needed in high dimensions. This yields an ultra-fast Gibbs sampling procedure, with each step of the Gibbs sampler requiring $O(n p)$ operations. Thus, computational effort for the filtering step is linear in both dimensions.

Step 2 fits a rescaled spike and slab model using only those variables that are not filtered in Step 1. Model fitting is implemented using a Gibbs sampler. Computational times are generally rapid as the number of variables at this point are a fraction of the original size, $p$. A blocking technique is used to further reduce computational times. The posterior mean of the regression coefficients, which we refer to as the BMA, is calculated and returned. This (restricted) BMA is used as an estimator for the regression coefficients.

Step 3 calculates the gnet. In the optimization, the gnet's $\ell_{2}$-regularization parameters are fixed (these being determined from the restricted BMA obtained in Step 2) and its solution path with respect to its $\ell_{1}$-regularization parameter is calculated using the lars R package (Hastie and Efron, 2007) [a package dependency of spikeslab]. The lars wrapper is called with type $=" 1 \mathrm{ar}$ " to produce the full LAR path solution (Efron et al., 2004). The gnet is defined as the model in this path solution minimizing the AIC criterion. Note importantly, that cross-validation is not used to optimize the $\ell_{1}$-regularization parameter. The gnet estimator is generally very stable, a property that it inherits from the BMA, and thus even simple model selection methods such as AIC work quite well in optimizing its path solution. This is different than say the elastic net (Zou and Hastie,
2005) where cross-validation is typically used to determine its regularization parameters (often this involves a double optimization over both the $\ell_{1}$ - and $\ell_{2}$-regularization parameters). This is an important feature which reduces computational times in big- $p$ problems.

\section{Low-dimensional settings}

Although the spike and slab algorithm is especially adept in high-dimensional settings, it can be used effectively in classical settings as well. In these lowdimensional scenarios when $p<n$, the algorithm is implemented by applying only Steps 2 and 3 (i.e., Step 1 is skipped). The default setting for spikeslab, in fact, assumes a low-dimensional scenario.

As illustration, we consider the benchmark diabetes data $(n=442, p=64)$ used in Efron et al. (2004) and which is an example dataset included in the package. The response $Y$ is a quantitative measure of disease progression for patients with diabetes. The data includes 10 baseline measurements for each patient, in addition to 45 interactions and 9 quadratic terms, for a total of 64 variables for each patient. The following code implements a default analysis:

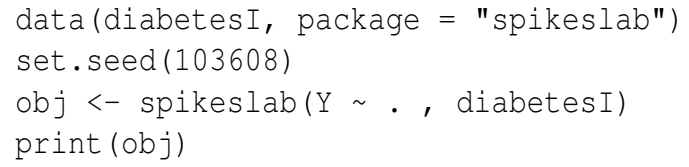

The print call outputs a basic summary of the analysis, including a list of the selected variables and their parameter estimates (variables selected are those having nonzero gnet coefficient estimates):

$\begin{array}{lrrrr} & \text { bma } & \text { gnet } & \text { bma.scale } & \text { gnet.scale } \\ \text { bmi } & 24.076 & 23.959 & 506.163 & 503.700 \\ \text { ltg } & 23.004 & 22.592 & 483.641 & 474.965 \\ \text { map } & 14.235 & 12.894 & 299.279 & 271.089 \\ \text { hdl } & -11.495 & -10.003 & -241.660 & -210.306 \\ \text { sex } & -7.789 & -6.731 & -163.761 & -141.520 \\ \text { age.sex } & 6.523 & 5.913 & 137.143 & 124.322 \\ \text { bmi.map } & 3.363 & 4.359 & 70.694 & 91.640 \\ \text { glu.2 } & 2.185 & 3.598 & 45.938 & 75.654 \\ \text { age.ltg } & 1.254 & 0.976 & 26.354 & 20.528 \\ \text { bmi.2 } & 1.225 & 1.837 & 25.754 & 38.622 \\ \text { age.map } & 0.586 & 0.928 & 12.322 & 19.515 \\ \text { age.2 } & 0.553 & 0.572 & 11.635 & 12.016 \\ \text { sex.map } & 0.540 & 0.254 & 11.349 & 5.344 \\ \text { glu } & 0.522 & 0.628 & 10.982 & 13.195 \\ \text { age.glu } & 0.417 & 0.222 & 8.757 & 4.677\end{array}$

In interpreting the table, we note the following:

(i) The first column with the heading bma lists the coefficient estimates for the BMA estimator obtained from Step 2 of the algorithm. These values are given in terms of the standardized covariates (mean of 0 and variance of 1 ). 
(ii) The second column with the heading gnet lists the coefficient estimates for gnet obtained from Step 3 of the algorithm. These values are also given in terms of the standardized covariates.

(iii) The last two columns are the BMA and gnet estimators given in terms of the original scale of the variables. These columns are used for prediction, while the first two columns are useful for assessing the relative importance of variables.

Note that all columns can be extracted from the spike and slab object, obj, if desired.

\section{Stability analysis}

Even though the gnet accomplishes the goal of variable selection, it is always useful to have a measure of stability of a variable. The wrapper cv.spikeslab can be used for this purpose.

The call to this wrapper is very simple. Here we illustrate its usage on the diabetes data:

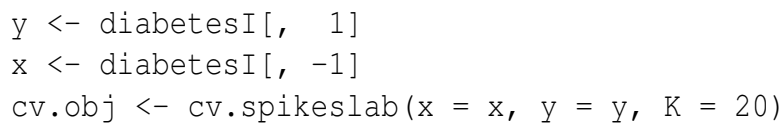

This implements 20-fold validation (the number of folds is set by using the option $\mathrm{K}$ ). The gnet estimator is fit using the training data and its test-set meansquared-error (MSE) for its entire solution-path is determined. As well, for each fold, the optimal gnet model is determined by minimizing test-set error. The average number of times a variable is selected in this manner defines its stability (this is recorded in percentage as a value from $0 \%-100 \%$ ). Averaging the gnet's test-set MSE provides an estimate of its MSE as a function of the number of variables.

The gnet's coefficient values (estimated using the full data) and its stability values can be obtained from the $\mathrm{Cv}$. obj using the following commands:

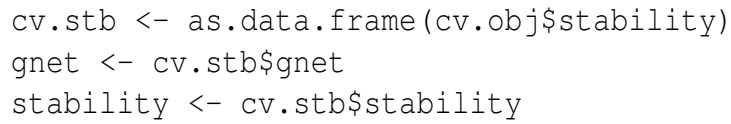

Figure 1 (top) plots the gnet's cross-validated MSE curve as a function of the model size. The plot was produced with the command

plot (cv.obj, plot.type $\left.=" c v^{\prime}\right)$

Close inspection (confirmed by considering the object, cv.obj) shows that the optimal model size is somewhere between 9 and 17, agreeing closely with our previous analysis. The bottom plot shows how gnet coefficient estimates vary in terms of their stability values (obtained by plotting gnet versus stability). There are 10 variables having stability values greater than $80 \%$.
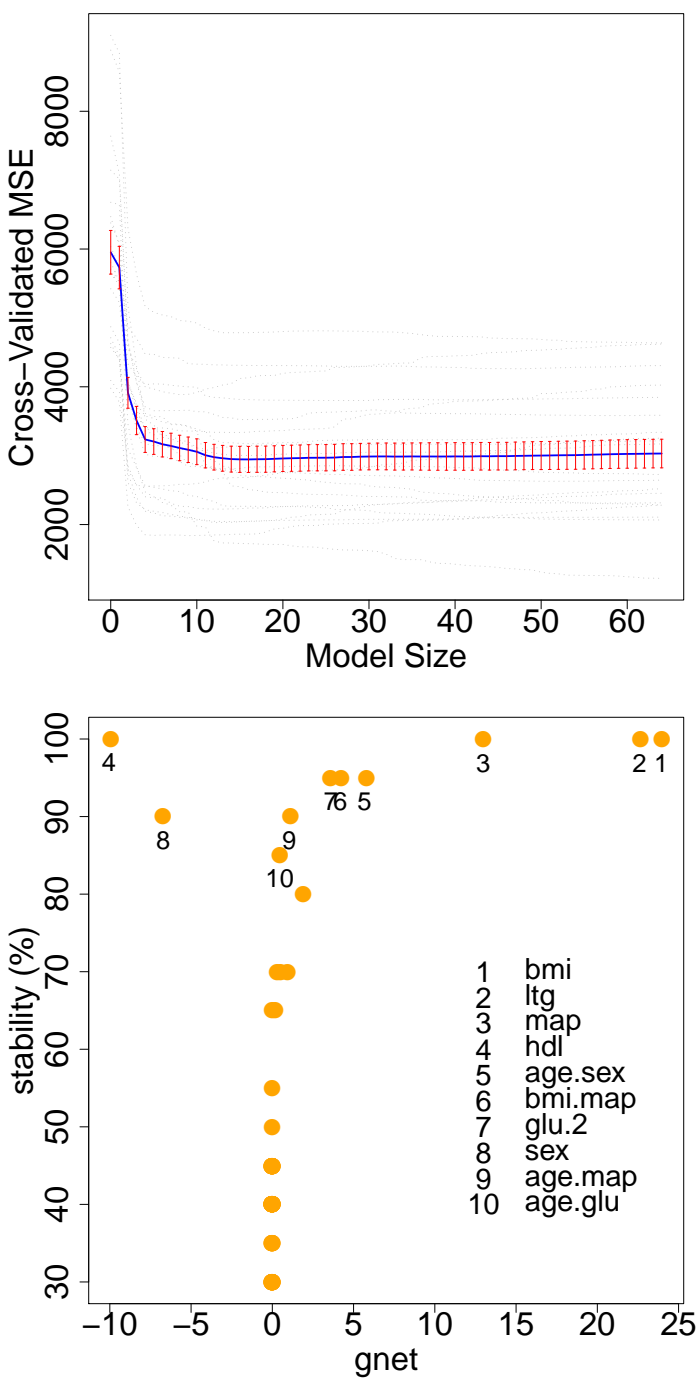

Figure 1: Stability analysis for diabetes data.

\section{High-dimensional settings}

To analyze $p \gg n$ data, users should use the option bigp. smalln=TRUE in the call to spikeslab. This will invoke the full spike and slab algorithm including the filtering step (Step 1) which is crucial to success in high-dimensional settings (note that $p \geq n$ for this option to take effect). This three-step algorithm is computationally efficient, and because the bulk of the computations are linear in $p$, the algorithm should scale effectively to very large $p$-problems. However, in order to take full advantage of its speed, there are a few simple, but important rules to keep in mind.

First, users should avoid using the formula and data-frame call to spikeslab when $p$ is large. Instead they should pass the $x$-covariate matrix and $y$ response vector directly. This avoids the tremendous overhead required to parse formula in $\mathrm{R}$.

Second, the final model size of the BMA and gnet are controlled by two key options; these must be set properly to keep computations manageable. These 
options are: bigp.smalln.factor and max.var. The first option restricts the number of filtered variables in Step 1 of the algorithm to be no larger than $F n$, where $F>0$ is the value bigp.smalln. factor. The default setting $F=1$ should be adequate in most scenarios [one exception is when $n$ is very large (but smaller than $p$ ); then $F$ should be decreased to some value $0<F<1$ ]. The second option, max.var, restricts the number of selected variables in both Steps 1 and 3 of the algorithm. Its function is similar to bigp.smalln. factor, although unlike bigp. smalln. factor, it directly controls the size of gnet. The default value is max.var $=500$. In most examples, it will suffice to work with bigp. smalln. factor.

Thus, if $x$ is the $x$-matrix, $y$ is the $y$-response vector, and $f$ and $m$ are the desired settings for bigp.smalln. factor and max.var, then a generic call in high-dimensional settings would look like:

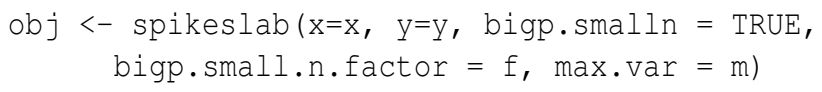

Although spikeslab has several other options, most users will not need these and the above call should suffice for most examples. However, if computational times are a still of concern even after tuning $f$ and $m$, users may consider changing the default values of $n$. iter 1 and $n$. iter2. The first controls the number of burn-in iterations used by the Gibbs sampler, and the second controls the number of Gibbs sampled values following burn-in (these latter values are used for inference and parameter estimation). The default setting is 500 in both cases. Decreasing these values will decrease computational times, but accuracy will suffer. Note that if computational times are not a concern, then both values could be increased to 1000 (but not much more is needed) to improve accuracy.

As illustration, we used a simulation with $n=$ 100 and $p=2000$. The data was simulated independently in blocks of size 40 . Within each block, the $x$-variables were drawn from a 50 -dimensional multivariate normal distribution with mean zero and equicorrelation matrix with $\rho=0.95$. With probability 0.9 , all regression coefficients within a block were set to zero, otherwise with probability 0.1 , all regression coefficients were set to zero except for the first 10 coefficients, which were each assigned a randomly chosen value from a standard normal distribution. Random noise $\varepsilon$ was simulated independently from a $\mathrm{N}\left(0, \sigma^{2}\right)$ distribution with $\sigma=0.4$.

The top plot in Figure 2 displays the path solution for the gnet. Such a plot can be produced by a call to the lars wrapper plot. lars using the gnet.obj obtained from the spikes lab call. As gnet.obj is a larstype object it is fully interpretable by the lars package, and thus it can be parsed by the packages' various wrappers. For convenience, the path solution can be produced by a direct call to plot; a typical call being:

obj <- spikeslab ( $x=x, y=y$, bigp.smalln = TRUE $)$ plot (obj, plot.type $=$ "path")

Actually Figure 2 was not produced by a call to plot but in fact was obtained by slightly modifying the plot.lars wrapper so as to display the paths of a variable color coded by its true coefficient value (blue for truly zero and red for truly nonzero). We did this in order to facilitate comparison to the lasso. The lasso path (obtained using the LAR-solution) is displayed in the bottom plot of Figure 2. Notice how in contrast to gnet, the path solution for the lasso has a wiggly "spaghetti"-like shape and that many of the truly nonzero coefficients are hard to identify because of this. This a direct consequence of the highcorrelation in the $x$-variables of this example. This correlation creates instability in the lasso-LAR solution, and this ultimately impacts its performance.
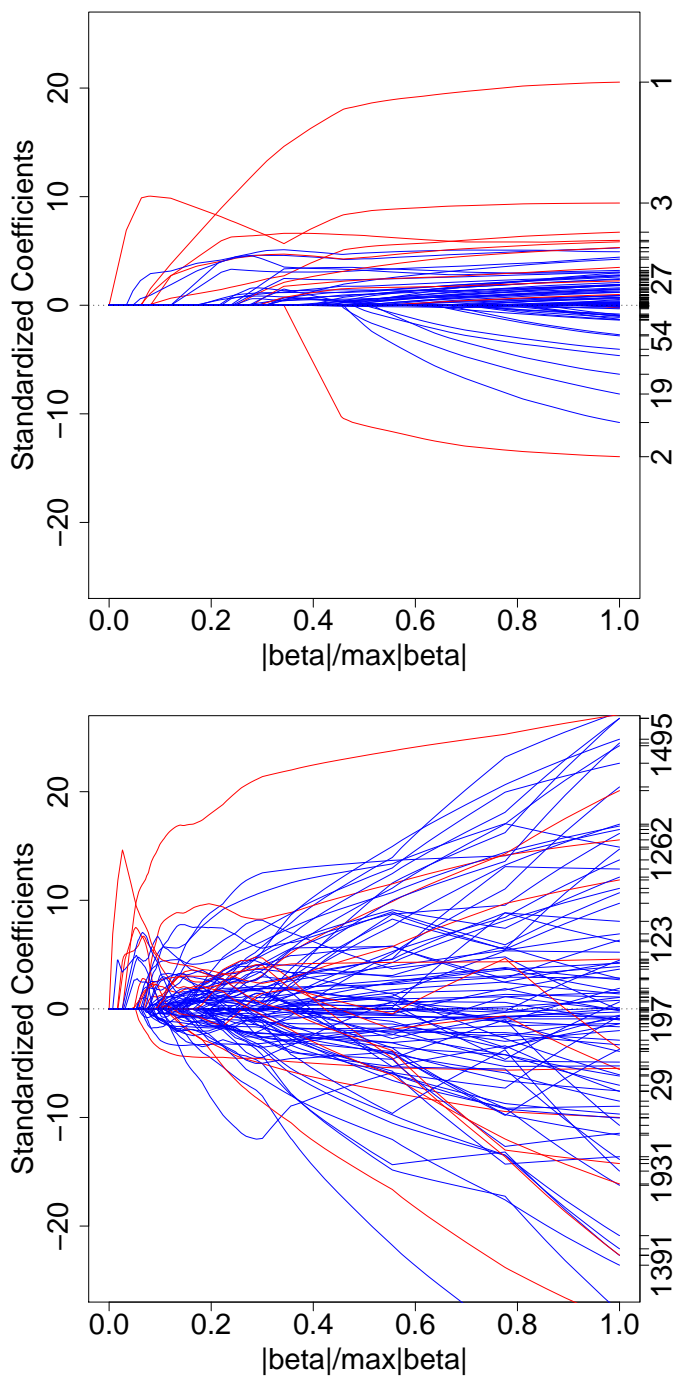

Figure 2: Path solutions for the gnet (top) and the lasso (bottom) from a correlated high-dimensional simulation $(n=100$ and $p=2000)$. Blue and red lines correspond to truly zero and truly nonzero coefficient values, respectively. 


\section{Summary}

The gnet incorporates the strength of Bayesian WGRR estimation with that of frequentist soft thresholding. These combined strengths make it an effective tool for prediction and variable selection in correlated high-dimensional settings. If variable selection is not of concern, and the key issue is accurate prediction, than the BMA may be preferred. Both the gnet and BMA can be computed using the spikeslab $\mathrm{R}$ package. This package is computationally efficient, and scales effectively even to massively large $p$-problems.

As one example of this scalability, we added 100,000 noise variables to the diabetes data set and then made a call to $\mathrm{cv}$.spikeslab with the added options bigp.smalln = TRUE, max.var $=100$ and parallel $=$ TRUE (as before we used $K=20$ fold validation). The parallel option invokes parallel processing that is implemented via the package snow (Tierney et al., 2008) [note that sending in an integer for the option parallel sets the number of socket clusters on the local machine on which the session is being initiated; in our example we actually used parallel $=8$ ]. The snow package should be loaded prior to making the cv.spikeslab call.

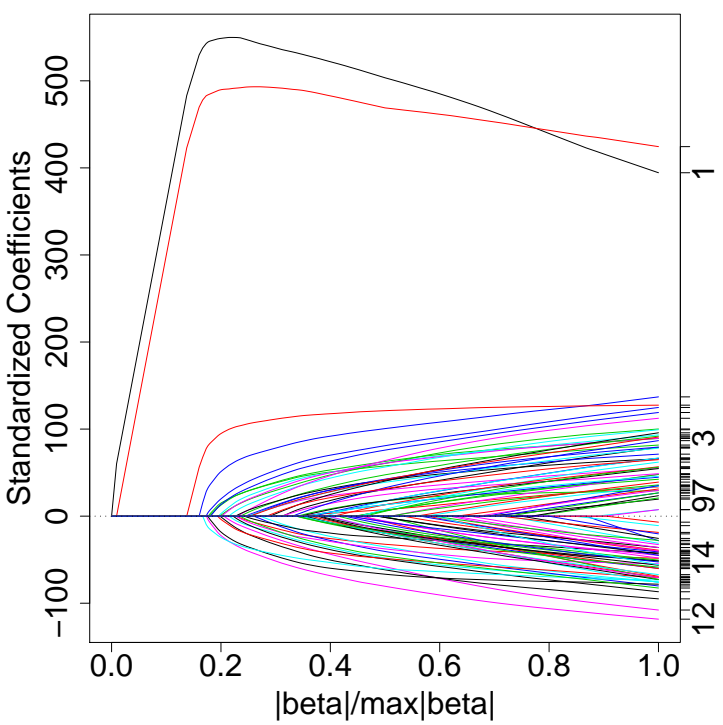

Figure 3: Path solution for the gnet for diabetes data with $p=100,000$ noise variables.

Figure 3 displays the gnet's path solution (obtained using the full data). While only 4 variables have path-profiles that clearly stand out, impressively these variables are the top 4 from our previous analysis. The gnet estimates (scaled to standardized covariates), its averaged cv-estimates, and the stability values for the top 15 variables were:

$\begin{array}{lrrr} & \text { gnet } & \text { gnet.cv } & \text { stability } \\ \text { ltg } & 20.1865498 & 20.14414604 & 100 \\ \text { bmi } & 18.7600433 & 22.79892835 & 100\end{array}$

$\begin{array}{lrrr}\text { map } & 4.7111022 & 4.83179363 & 95 \\ \text { hdl } & -2.5520177 & -2.66839785 & 95 \\ \text { bmi.2 } & 3.6204750 & 0.46308305 & 40 \\ \text { x.61746 } & 0.0000000 & -0.74646210 & 35 \\ \text { x.42036 } & 4.8342736 & 0.41993669 & 30 \\ \text { x.99041 } & 0.0000000 & -0.70183515 & 30 \\ \text { x.82308 } & 5.2728011 & 0.75420320 & 25 \\ \text { glu } & 1.3105751 & 0.16714059 & 25 \\ \text { x.46903 } & 0.0000000 & -0.65188451 & 25 \\ \text { x.57061 } & 0.0000000 & 0.73203633 & 25 \\ \text { x.99367 } & -2.7695621 & -0.22110463 & 20 \\ \text { tch } & 0.2542299 & 0.14837708 & 20 \\ \text { x.51837 } & 0.0000000 & -0.09707276 & 20\end{array}$

Importantly, note that the top 4 variables have greater than or equal to $95 \%$ stability (variables starting with "x." are noise variables). It is also interesting that 3 other non-noise variables, "bmi.2", "glu", and "tch" were in the top 15 variables. In fact, when we inspected the 100 variables that passed the filtering step of the algorithm (applied to the full data), we found that 10 were from the original 64 variables, and 6 were from the top 15 variables from our earlier analysis. This demonstrates stability of the filtering algorithm even in ultra-high dimensional problems.

Finally, we remark that in illustrating the spikeslab package in this article, we focused primarily on the spikeslab wrapper, which is the main entry point to the package. Other available wrappers include predict.spikeslab for prediction on test data, and sparsePC. spikeslab. The latter implements variable selection for multiclass gene expression data (Ishwaran and Rao, 2010).

In future work we plan to extend the rescaled spike and slab methodology to high-dimensional generalized linear models. At that time we will introduce a corresponding wrapper.

\section{Acknowledgements}

This work was partially funded by the National Science Foundation grant DMS-0705037. We thank Vincent Carey and two anonymous referees for helping to substantially improve this manuscript.

\section{Bibliography}

T.T. Cai. and J. Lv. Discussion: The Dantzig selector: statistical estimation when $\mathrm{p}$ is much larger than $\mathrm{n}$. Ann. Statist., 35:2365, 2007.

B. Efron, T. Hastie, I. Johnstone and R. Tibshirani. Least angle regression (with discussion). Ann. Statist., 32:407-499, 2004.

J. Fan and J. Lv. Sure independence screening for ultra-high dimensional feature space. J. Royal Statist. Soc. B, 70(5):849-911, 2008. 
E.I. George and R.E. McCulloch. Variable selection via Gibbs sampling. J. Amer. Stat. Assoc., 88:881$889,1993$.

T. Hastie and B. Efron. lars: Least Angle Regression, Lasso and Forward Stagewise, 2010. R package version 0.9-7.

A.E. Hoerl and R.W. Kennard. Ridge regression: Biased estimation for nonorthogonal problems. Technometrics, 12:55-67, 1970.

A.E. Hoerl and R.W. Kennard. Ridge regression: Applications to nonorthogonal problems (Corr: V12 p723). Technometrics, 12:69-82, 1970.

H. Ishwaran and J.S. Rao. Spike and slab variable selection: frequentist and Bayesian strategies. Ann. Statist., 33:730-773, 2005.

H. Ishwaran and J.S. Rao. Generalized ridge regression: geometry and computational solutions when p is larger than n. Manuscript, 2010.

H. Ishwaran, U.B. Kogalur and J.S. Rao. spikeslab: Prediction and variable selection using spike and slab regression, 2010. R package version 1.1.2.

F. B. Lempers. Posterior Probabilities of Alternative Linear Models. Rotterdam University Press, Rotterdam, 1971.
T.J. Mitchell and J.J. Beauchamp. Bayesian variable selection in linear regression. J. Amer. Stat. Assoc., 83:1023-1036, 1988.

L. Tierney, A. J. Rossini, N. Li and H. Sevcikova snow: Simple Network of Workstations, 2008. R package version $0.3-3$

H. Zou and T. Hastie. Regularization and variable selection via the elastic net. J. Royal Statist. Soc. B, 67(2):301-320, 2005.

Hemant Ishwaran

Dept. Quantitative Health Sciences

Cleveland Clinic

Cleveland, $\mathrm{OH} 44195$

hemant.ishwaran@gmail.com

Udaya B. Kogalur

Dept. Quantitative Health Sciences, Cleveland Clinic

Cleveland, $\mathrm{OH} 44195$

kogalurshear@gmail.com

\section{J. Sunil Rao}

Dept. of Epidemiology and Public Health School of Medicine, University of Miami

Miami, FL 33136

rao.jsunilegmail.com 